\title{
Role of the Hedgehog pathway in hepatocellular carcinoma (Review)
}

\author{
XIN ZHENG ${ }^{1}$, WEN ZENG $^{2}$, XIAOHONG GAI ${ }^{1}$, QIURAN XU ${ }^{1}$, CHAO LI $^{1}$, \\ ZHEYONG LIANG $^{1}$, HANG TUO ${ }^{1}$ and QINGGUANG LIU ${ }^{1}$ \\ ${ }^{1}$ Department of Hepatobiliary Surgery, The First Affiliated Hospital of Xi'an Jiaotong University; \\ ${ }^{2}$ Department of General Surgery, Xi'an Central Hospital, Xi'an, Shaanxi 710061, P.R. China
}

Received June 20, 2013; Accepted August 2, 2013

DOI: $10.3892 /$ or. 2013.2690

\begin{abstract}
The Hedgehog (Hh) pathway is an evolutionarily conserved signaling mechanism that controls many aspects of cell differentiation and the development of tissues and organs during embryogenesis. Early investigations have focused on the effects of $\mathrm{Hh}$ activity on the development of organs including skin, gut, the nervous system and bone. However, in addition to normal developmental processes, these investigations also found that Hh signaling is involved in aberrant proliferation and malignant transformation. Consequently, the role of $\mathrm{Hh}$ in cancer pathology, and its modulation by environmental factors is the subject of many investigations. Numerous environmental toxins, alcohol, and hepatitis viruses can cause hepatocellular carcinoma (HCC), which is the most common form of liver cancer. Significant hyperactivation of $\mathrm{Hh}$ signaling has been observed in liver injury and cirrhosis which often leads to the development of HCC lesions. Moreover, Hh activity plays an important role in the progression of HCC. Here, we review findings relevant to our understanding of the role of $\mathrm{Hh}$ signaling in HCC pathogenesis.
\end{abstract}

Correspondence to: Professor Qingguang Liu, Department of Hepatobiliary Surgery, The First Affiliated Hospital of Xi'an Jiaotong University, 277 Yanta West Road, Xi'an, Shaanxi 710061 P.R. China

E-mail: xin.zheng.xjtu@gmail.com

Abbreviations: HCC, hepatocellular carcinoma; Hh, Hedgehog; TACE, transcatheter arterial chemoembolization; RFA, radiofrequency ablation; EMT, epithelial-mesenchymal transition; Shh, Sonic hedgehog; Ihh, Indian hedgehog; Dhh, Desert hedgehog; PTCH, Patched; Smo, Smoothened; Cos 2, kinesin-like protein Costal 2; Fu, fused; SuFu, suppressor of fused; BCC, basal cell carcinoma; Hip, hedgehog interacting protein; MF, myofibroblast; LOH, loss of heterozygosity; HBx, hepatitis B virus encoded X protein; NBCCS, Nevoid basal cell carcinoma syndrome

Key words: Hedgehog, cell signal, hepatocellular carcinoma, targeting therapy

\section{Contents}

1. Introduction

2. Characterization of Hh signaling

3. Aberrant activation of $\mathrm{Hh}$ signaling in cancers

4. The role of Hh signaling in $\mathrm{HCC}$

5. Hh target therapies for cancer

6. Conclusions

\section{Introduction}

Hepatocellular carcinoma (HCC) is a global health problem. $\mathrm{HCC}$ is one of the most common forms of cancer and the third leading reason for cancer-related death (1). Unfortunately, there are no typical clinical manifestations of early-stage HCC. Therefore, most patients are identified at later stages of the disease, and are often treated by therapies including radical liver resection and liver transplantation. The long-term outcome of current palliative treatments for HCC such as transcatheter arterial chemoembolization (TACE) and radiofrequency ablation (RFA) is not satisfactory $(2,3)$. Thereby, there is an urgent need to investigate the underlying molecular pathogenesis in order to develop novel therapies for HCC.

Hedgehog $(\mathrm{Hh})$ signaling pathways are objects of intense investigation in HCC studies. Hh signaling contributes to cell differentiation, organ formation, carcinogenesis and cancer metastasis. Studies indicate that $\mathrm{Hh}$ signaling is aberrantly activated to promote proliferation, viability, migration and invasion of HCC cells (4-6). In this review, we summarize basic components of the Hh pathway and its role in $\mathrm{HCC}$ pathogenesis.

\section{Characterization of Hh signaling}

Hh signaling was first identified by the Nobel laureates Wieschaus and Nusslein-Volhard by mutagenesis screening assays in Drosophila (7). They found that Hh mutations lead to abnormal hedgehog-like denticle formation in flies. This signaling pathway is conserved from flies to vertebrates, and includes 7 main components: Hedgehog (Hh), Patched $(\mathrm{PTCH})$, Smoothened (Smo), GLIs, kinesin-like protein Costal 2 (Cos 2), fused (Fu) and suppressor of fused (SuFu). 


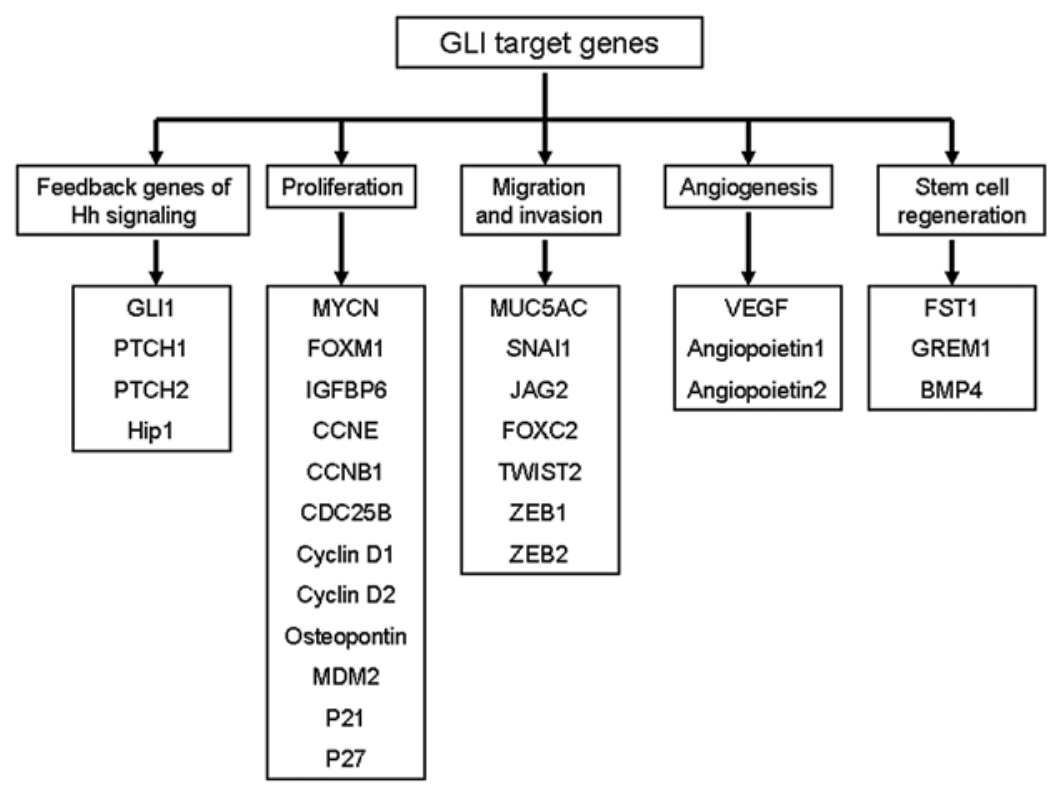

Figure 1. The GLI target genes.

There are 3 types of $\mathrm{Hh}$ protein found in mammalians including Sonic hedgehog (Shh), Indian hedgehog (Ihh) and Desert hedgehog (Dhh) (8). The Hh protein precursor consists of a C-terminal protease domain and an N-terminal signaling unit. After undergoing a series of modifications, the N-terminus of the Hh protein is modified with palmitates (9), and its C-terminus is bound to a cholesterol moiety (10), which facilitates $\mathrm{Hh}$ protein to be secreted from cells.

$\mathrm{Hg}$ targets the $\mathrm{PTCH}$ receptor, which is a 12-span transmembrane protein. PTCH acts as a negative regulator of the Hh pathway by inhibiting the $\mathrm{G}$ protein-coupled receptor Smo in the absence of Hh protein. After binding with Hh protein, PTCH allows Smo to release GLIs from a multiple protein complex. These GLIs then enter the cell nucleus to regulate transcription of target genes. There are three GLI proteins found in humans including GLI1, GLI2 and GLI3. The GLI1 gene was initially identified as being amplified in human glioma (11-13).

GLI1, GLI2 and GLI3 share a highly similar protein structure consisting of 5 conserved tandem zinc fingers, a relative conserved N-terminal domain, several possible PKA sites and other small conserved domains in the C-terminus. They also have a common DNA-binding domain which can target the DNA sequence GACCACCCA (14). As shown in Fig. 1, several GLI target genes have been identified which affect cell proliferation [MYCN (15), CCND1 (16), CCND2 (17), FOXM1 (18)], stem regeneration [JAG2 (16), FST (19)], cell survival [BCL2 (16), CFLAR (20)], and EMT [FOXC2 (21), SNAI1 (4), TWIST2 (22)].

GLI proteins associate with $\mathrm{Cos} 2, \mathrm{Fu}$ and $\mathrm{SuFu}$ to join the GLI-Cos 2-Fu-SuFu complex in the cytoplasm in the absence of Hh (23). While associated with the cytoplasmic complex GLI proteins are sequestered from the nucleus and target genes are not activated. In addition, protein kinases including PKA, GSK3 and CK1 can promote phosphorylation of GLIs to suppress there transcriptional activity. These phosphorylated GLIs enter the cell nucleus to inhibit the regulatory effect of
Hh signaling on target genes by SIN3-HDAC or SKI-HDAC dependent mechanisms (24).

When Hh protein binds with PTCH, Smo is activated and translocates to primary cilia. Consequently, GLI proteins are cleaved into activated forms, released from the cytoplasmic GLI-Cos 2-Fu-SuFu complex, and bind to promoter regions to regulate the transcription of downstream genes. However, recent studies indicate that GLI activation can also be controlled by Hh-independent mechanisms. For example, the RAS-MEK/AKT pathway can modify the Hh pathway in cancer cells (25). Other studies suggest that TGF $\beta 1$ can induce GLI1 upregulation in HCC (4).

\section{Aberrant activation of Hh signaling in cancers}

Components of the Hh pathway are frequently mutated in cancer. There have been three main events by which the Hh pathway is aberrantly activated in cancer (Fig. 2). Type I events underlie a ligand-independent constitutive activation of $\mathrm{Hh}$ signaling due to mutations that inactivate negative regulators including PTCH or SuFu, and/or hyperactivation of Smo and GLI1, which are found in basal cell carcinoma (BCC) (26) and medulloblastoma (27). Type II events lead to ligand-dependent autocrine signaling in which cancer cells produce and secrete Hh ligands, which have been found in lung cancer (28), gastrointestinal cancer (29) and prostate cancer (30). Type IIIa events cause ligand-dependent paracrine affects by activating Hh signaling in stromal cells as a result of $\mathrm{Hh}$ ligands excreted by cancer cells, mostly in pancreatic cancer (31). Type IIIb events arise as a ligand-dependent reverse paracrine response in which the Hh pathway of cancer cells is activated by $\mathrm{Hh}$ ligands excreted by stromal cells, which are found in malignant lymphoma and plasmacytoma (32).

Hh signaling contributes to the pathogenesis of various cancers. For example, GLI1 was found to be highly amplified in human glioblastoma tissues and derived cell lines and is attributed to gliomagenesis (33). Aberration of Hh signaling 


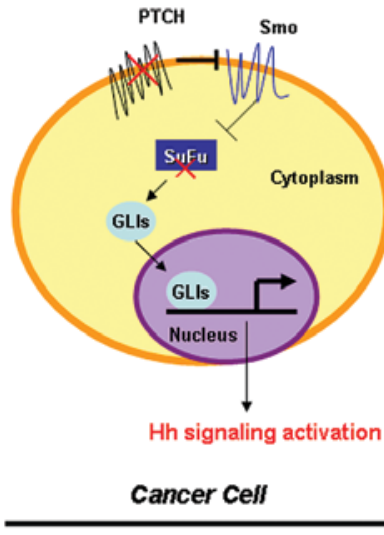

Type I

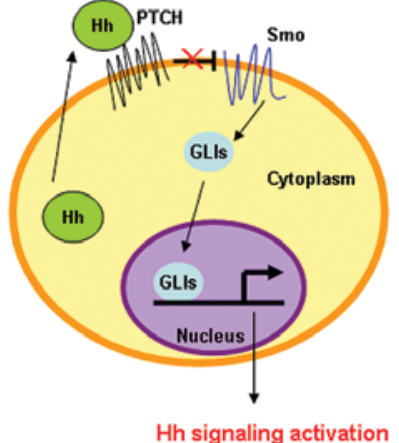

Cancer Cell

Type II

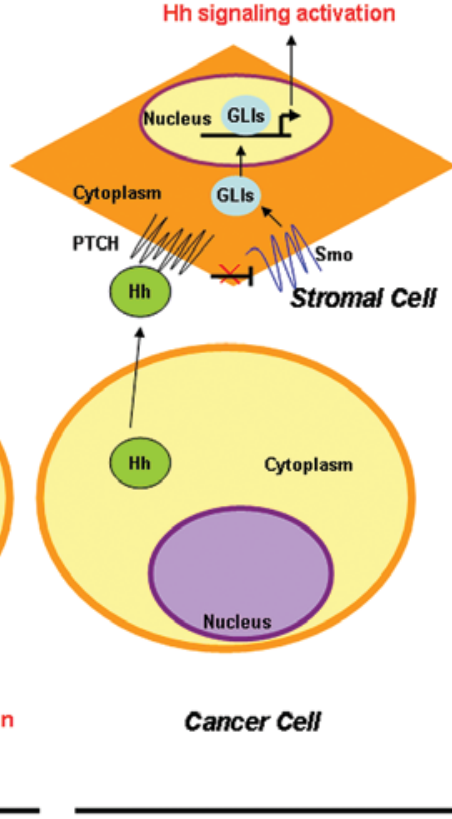

Type IIIa

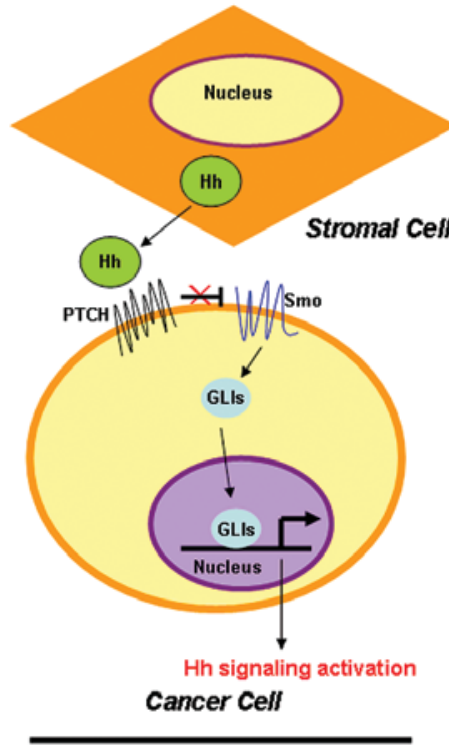

Type IIb

Figure 2. A model of various types of aberrant activation of the Hh pathway in cancer. Hh, Hedgehog.

was also found in medulloblastoma which is the leading malignant pediatric brain tumor worldwide.

Experiments demonstrate that aberrant activation of $\mathrm{Hh}$ signaling promotes brain tumor growth via upregulation of cell proliferation (34). It has become clear that a majority of basal cell carcinoma (BCC) patients have aberrant activation of the Hh pathway (35). Consistently, there is often constitutive activation of downstream targets of the Hh pathway in BCC. For example, Watkins et al (36) found that Shh factors are overexpressed in small-cell lung cancer (SCLC). Furthermore, the Hh pathway in lung cancer can be activated in a Type IIIa manner by an Hh ligand-dependent paracrine mechanism.

Lauth et al (37) report that $\mathrm{Hh}$ signaling of stromal cells in lung cancer can be activated by Shh secreted by cancer cells, and that activated stromal cells secrete numerous cytokines to promote the malignant phenotype of lung cancer cells. In addition, constitutive Hh signaling has also been identified as a critical mechanism leading to breast cancer (38). Various mutations of $\mathrm{Hh}$ signaling, including inactivating mutations of PTCH, activating missense mutations of Smo, and loss of function mutations of $\mathrm{SuFu}$ are found in breast cancer and are implicated in mammary carcinoma development $(39,40)$.

In addition to mutations, epigenetic regulatory mechanisms can lead to activation of Hh signaling. These include hypermethylation of the promoters of hedgehog interacting protein (Hip) and PTCH, and hypomethylation of Shh promoters (41). Aberrant Hh signaling has been found in colon cancer, and appears to promote the progression of colon cancer from local adenoma in colon epithelium to advanced adenoma with distant metastasis (42). Multiple mechanisms mediated by ligand-dependent and ligand-independent signaling cause hyperactivation of $\mathrm{Hh}$ signaling in colon cancer. For example, overexpression of Shh has been found in colon cancer tissues (42), while both loss-of-function PTCH mutations and gain-of-function Smo mutations are frequently found in colon cancers (43). Indeed, aberrant activation of Hh signaling has been shown to promote proliferation, migration, and invasion of colon cancer cells $(44,45)$.

\section{The role of Hh signaling in HCC}

As mentioned above, the Hh pathway is altered and contributes to the development and progression of many types of cancer. These include liver cancer, prostate cancer, neuroblastoma and ovarian cancer. The role of $\mathrm{Hh}$ in $\mathrm{HCC}$ is particularly compelling.

Many groups have found aberrant activation of $\mathrm{Hh}$ signaling in HCC $(4,6,46)$. However, the underlying mechanisms of Hh activation in HCC are complex. Lu et al (46) reported that Shh treatment at a concentration of $0.5 \mu \mathrm{g} / \mathrm{ml}$ increased GLI1 expression and promoted HCC cell invasion and migration. These results indicate that $\mathrm{Hh}$ signaling in HCC can be activated by a ligand-dependent manner. Others have also found that Shh treatment can stimulate Hh signaling in HCC cells (47).

Interestingly, Sicklick et al (48) found overexpression of Smo and an increase in the stoichiometric ratio of Smo to PTCH mRNA levels in HCC, and that this effect is related with tumor size and may be a prognostic marker of liver cancer. Sicklick et al (48) also found mutant Smo expression in Hep3B cells and that cyclopamine (an inhibitor against wild-type Smo) did not affect Hh signaling activation and growth of these cells. However, after treatment with KAAD-cyclopamine, which is a blocker of mutant Smo, Hh signaling activity in Hep3B was repressed by $\sim 50 \%$ and the growth rate was decreased by $94 \%$.

Tada et al (49) found hypermethylation of Hip promoters and loss of heterozygosity (LOH) at the Hip locus, which was attributed to downregulation of Hip in HCC tissues. Recent 
experiments also found that HCC cells secrete Shh to induce glycolysis of neighboring MFs, which consequently leads to the production of myofibroblast-derived lactate that HCC cells use as an energy source (50). These results indicate that $\mathrm{Hh}$ ligand-dependent paracrine manner (Type IIIa) may play an important role in the pathogenesis of HCC.

As shown in Fig. 3, several studies revealed the key effects of Hh signaling on HCC. Previous studies have found that GLI1 expression is positively correlated with the EMT phenotype and with intrahepatic metastasis and portal venous invasion of human HCCs (6). In addition, gene expression microarray analysis found that GLI1 mRNA overexpression in HCC tissues was associated with rapid recurrence of HCC tumors after surgery (4). Other studies used immunohistochemistry to find that GLI1 expression in HCC tissues is associated with disease-free survival and overall survival. In contrast, in vitro experiments indicated that forced expression of GLI1 promotes proliferation, viability, colony formation, migration and invasion of Huh7 cells, while silencing GLI1 expression in SNU398 cells produced opposite results.

To elucidate the mechanisms that may underlie the effects of GLI1 on HCC growth and motility, we searched the sequence of the SNAI1 promoter to find potential GLI1 protein binding sites. Through chromatin immunoprecipitation (ChIP) assays, GLI1 was found to bind to a region in the SNAI1 promoter located -1,417/-1,214 bp upstream of the transcriptional start site. Additionally, overexpression of GLI1 induced upregulation of SNAI1 in Huh7 cells and knockdown of GLI1 in SNU398 cells decreased the expression of SNAI1. Consistently, the EMT phenotype of Huh7 cells was induced by upregulation of GLI1, and knockdown of GLI1 reversed EMT to MET in SNU398 cells. These results strongly indicate that GLI1 overexpression is essential for HCC cells to obtain and maintain their EMT phenotype. On the other hand, we also found that TGF $\beta 1$ treatment leads to upregulation of GLI1, which is necessary for TGF $\beta 1$-driven EMT in Huh7 cells. During investigation of the anti-HCC function of sulfatase 2 inhibitor OKN-007, we found that activity of Hh signaling was modulated by sulfatase 2 in HCC cells (3).

Hepatitis B virus encoded X protein (HBx) can also contribute to the pathogenesis of HCC. Arzumanyan et al (51) found a positive relationship between HBx and Hh signaling components in HCC cell lines, samples of HCC secondary to HBV infection and relevant mouse specimens. Inhibiting $\mathrm{Hh}$ signaling eliminated the ability of HBx to promote cell migration, anchorage-independent growth, and tumor development of HCC. Hence, it seems that activation of Hh signaling is necessary for $\mathrm{HBx}$ to accelerate hepatocarcinogenesis.

Pereira et al (52) found increased hepatic expression of Hh ligands in all patients with chronic hepatitis during liver cirrhosis and HCC. In addition, inhibiting Hh signaling in these Hh-responsive cells blocked fibrosis. Based on these findings, it seems that hepatitis infection can increase the production of Hh ligands in hepatocytes and liver accumulation of Hh-responsive cells, which aggravates liver cirrhosis and hepatocarcinogenesis.

Autophagy plays a central role in controlling apoptosis of HCC cells. Wang et al (47) found that activating the Hh pathway through treatment of Shh and its agonists (SAG and purmorphamine) blocked the induction of autophagy in several HCC cell lines. In addition, inhibition of Hh signaling by GANT61, which is a small-molecule inhibitor of GLI1, induced autophagy. These results demonstrate that $\mathrm{Hh}$ signaling is involved in aberrant autophagy of HCC cells.

Activation of $\mathrm{Hh}$ signaling was also found to promote the invasion and metastasis of HCC via mediating the ERK-driven overexpression of MMP-9 (46). EMT is believed to be the critical event to promote migration and invasion of cancer cells. Our preliminary clinical data indicate that activation of Hh signaling is correlated positively with the mesenchymal marker S100a4 and clinicopathological characteristics indicative of an enhanced metastatic potential of HCC (6), and negatively correlated with expression of epithelial marker E-cadherin. In addition, forced expression of GLI1 was found to trigger HCC EMT in vitro. Experiments utilizing Mdr2 knockout mice provide convincing evidence of an important role of Hh signaling in the development of HCC from liver cirrhosis (53). These experiments found that $\mathrm{Hh}$ signaling was activated aberrantly in Mdr2 knockout mice compared with wild-type mice. Chen et al (54) reported that well-differentiated CD133(+)/ALDH(high) or CD133(+)/EpCAM(+) HCC cells (Huh7 and Hep3B cells) displayed features similar to HCC stem cells. These cancer stem-like cells were found to have enhanced Hh signaling activity which was responsible for their chemoresistance and tumor invasion.

\section{Hh target therapies for cancer}

The first Hh signaling inhibitor, cyclopamine, was found by Cooper et al (55) in 1998. Since then, other small-molecule Hh signaling inhibitors have been designed for in vitro and in vivo studies of cancer treatment (briefly summarized in Fig. 4). These inhibitors target different components of $\mathrm{Hh}$ signaling including Smo, Shh and GLI1 (56). Some of these compounds have been tested in clinical trials; for example, vismodegib/GDC-0449, LED225 and GANT61.

Vismodegib, also named GDC-0449, is a small molecule designed by Curis Genentech to target Smo. The antitumor effect of vismodegib was first identified in a medulloblastoma allograft mouse model, in which vismodegib treatment at doses of $12.5 \mathrm{mg} / \mathrm{kg}$ BID repressed the growth of medulloblastoma completely (57). Preclinical studies also found similar antitumor effects of vismodegib on colon cancer, pancreatic cancer, lung cancer, esophageal cancer and gastric cancer (58-60). These experiments found that GDC-0449 represses cell growth and promotes the apoptosis of pancreatic cancer cells by activating TRAIL-R/DR signaling, caspase-3 signaling, and inducing PARP cleavage (61).

Most clinical trials of vismodegib have been carried out in BCCs and ovarian cancers. In a phase I clinical trial of BCCs, vismodegib showed a $55 \%$ response rate in 33 advanced BCC patients and was well tolerated (62). The most common side-effects included muscle spasms, altered taste, weight loss and hyponatremia.

Phase II clinical trials of vismodegib were performed in advanced ovarian cancer and BCCs. These clinical trials on advanced ovarian cancer conducted by Genentech found that the average time for the disease to progress was 7.5 months in the vismodegib group compared with 5.8 months in the placebo group (63). Phase II clinical trials of vismodegib on 


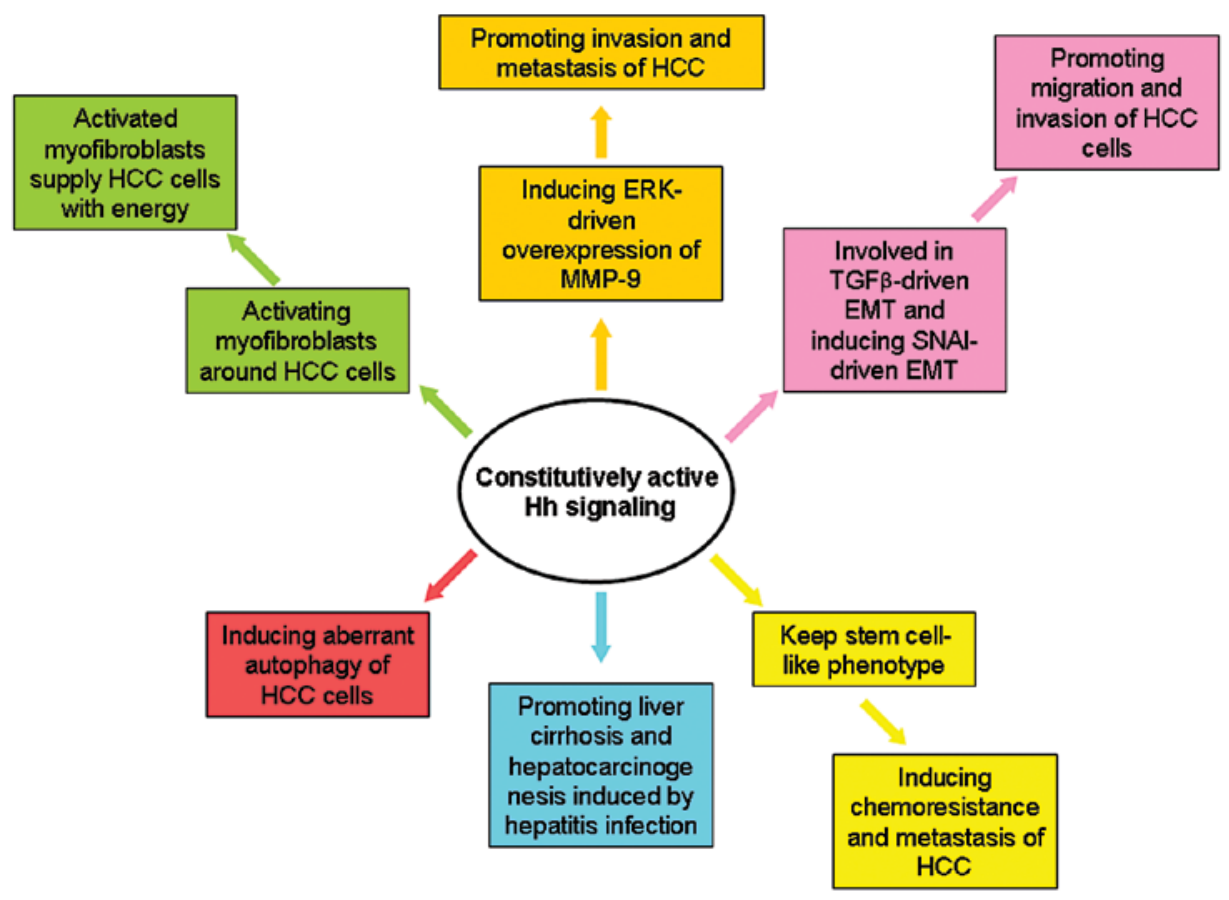

Figure 3. The different molecular mechanisms by which constitutively active $\mathrm{Hh}$ signaling induce hepatocarcinogenesis and promote the malignant phenotypes of HCC. Hh, Hedgehog; HCC, hepatocellular carcinoma.

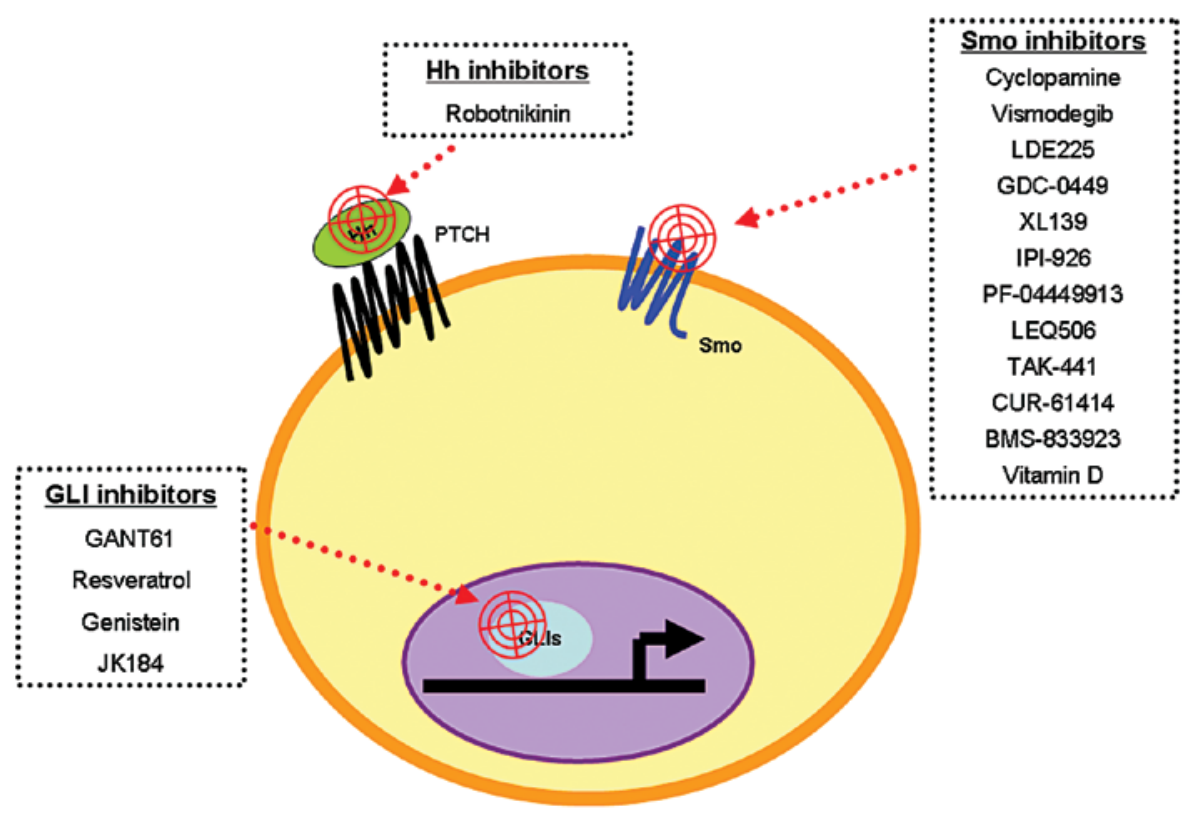

Figure 4. The small molecules against Hh signaling. Hh, Hedgehog.

BCCs found an objective response rate in metastatic basal cell carcinoma that reached up to $30.3 \%$, and an objective response rate in locally advanced basal cell carcinoma of $42.9 \%$ (64). On the basis of these clinical trials (65), the US Food and Drug Administration approved the use of Erivedge ${ }^{\mathrm{TM}}$ (vismodegib) capsules for the treatment of patients with recurrent, locally advanced or metastatic BCC in January 30, 2012.

LDE225 is another inhibitor of Smo designed by Novartis. Preclinical experiments found that it suppresses the development of BCC. Novartis conducted 2 clinical trials of LDE225 for cancer therapy using different methods of administration. An LDE225 cream was used to treat nevoid basal cell carcinoma syndrome (NBCCS) in one phase I clinical trial which found a $92.3 \%$ (12/13) clinical response rate and a mean volume reduction of $49.8 \%$ in the LDE225 group compared to $9.1 \%$ in the placebo group (66). Interestingly, Hh signaling in NBCCS tissues was also inhibited by this treatment.

Another phase I clinical trial was carried out to test an oral dosing regimen of LDE225 in patients with advanced solid tumors. As expected, GLI1 mRNA was reduced when the 
dose of LDE225 was increased. Side-effects included fatigue, nausea, vomiting, anorexia, muscle cramps and dysgeusia (67). Unfortunately, apparent drug-resistance was observed in these clinical trials, which thwarted its clinical development. This drug-resistance was considered to be associated with overexpression of Gli2 and point mutations in Smo, which maintained constitutive activation of $\mathrm{Hh}$ signaling in solid tumors.

GANT61 is a hexahydropyrimidine derivative designed as a small molecular inhibitor that targets GLI1. Lauth et al (68) first identified its ability to reduce GLI1-mediated transcription and named it GANT61 (for Gli-ANTagonist). Although it has not yet been tested in clinical trials, GANT61 has displayed promising antitumor effects in several preclinical studies. GANT-61 was found to inhibit cell viability, spheroid formation, and GLI-DNA binding and transcriptional activities in pancreatic cancer stem cells (69). Moreover, GANT61 was found to induce autophagy, apoptosis and cytotoxicity in HCC cells in vitro and can inhibit tumor growth in SCID mice (47). Because of its favorable antitumor effect in a variety of cancers, this compound may serve as a potential targeting therapy against Hh signaling.

\section{Conclusions}

In normal adult liver tissues, Hh signaling is inactivated. However, constitutive activation of $\mathrm{Hh}$ signaling is found in lesions from chronic liver injuries and cirrhosis to HCC. Evidence demonstrates that alterations of $\mathrm{Hh}$ signaling promote HCC development and progression via different mechanisms. There are several small-molecule drugs specifically designed to target $\mathrm{Hh}$ signaling that are undergoing clinical trials. Some of these have been approved to treat BCC in the clinic. It is likely that targeting Hh signaling will develop into an important part of a comprehensive strategy to combat advanced HCCs and bring a brighter future to liver cancer patients.

\section{Acknowledgements}

This study was supported by grants from the National Natural Scientific Foundation of China (nos. 81272645 and 81072052 to Q.L), the Research Fund for the Doctoral Program of High Education of China from the Ministry of Education (no. 20120201120090 to X.Z.), and the Fundamental Research Funds for the Basic Research Operating Expenses Program of Central College sponsored by Xi'an Jiaotong University to X.Z. The authors thank Professor Gary S. Goldberg (Rowan University, NJ, USA) for the technical assistance.

\section{References}

1. Yang JD and Roberts LR: Hepatocellular carcinoma: a global view. Nat Rev Gastroenterol Hepatol 7: 448-458, 2010.

2. Sandhu DS, Tharayil VS, Lai JP and Roberts LR: Treatment options for hepatocellular carcinoma. Expert Rev Gastroenterol Hepatol 2: 81-92, 2008.

3. Zheng X, Gai X, Han S, et al: The human sulfatase 2 inhibitor 2,4-disulfonylphenyl-tert-butylnitrone $(\mathrm{OKN}-007)$ has an antitumor effect in hepatocellular carcinoma mediated via suppression of TGFB1/SMAD2 and Hedgehog/GLI1 signaling. Genes Chromosomes Cancer 52: 225-236, 2013.

4. Zheng X, Vittar NB, Gai X, et al: The transcription factor GLI1 mediates TGF $\beta 1$ driven EMT in hepatocellular carcinoma via a SNAI1-dependent mechanism. PLoS One 7: e49581, 2012.
5. Xu QR, Zheng X, Zan XF, Yao YM, Yang W and Liu QG: Glil expression and its relationship with the expression of Shh, Vimentin and E-cadherin in human hepatocellular carcinoma. Xi Bao Yu Fen Zi Mian Yi Xue Za Zhi 28: 536-539, 2012 (In Chinese).

6. Zheng X, Yao Y, Xu Q, Tu K and Liu Q: Evaluation of gliomaassociated oncogene 1 expression and its correlation with the expression of sonic hedgehog, E-cadherin and S100a4 in human hepatocellular carcinoma. Mol Med Rep 3: 965-970, 2010.

7. Nüsslein-Volhard C and Wieschaus E: Mutations affecting segment number and polarity in Drosophila. Nature 287: 795-801, 1980.

8. Heretsch P, Tzagkaroulaki L and Giannis A: Modulators of the hedgehog signaling pathway. Bioorg Med Chem 18: 6613-6624, 2010.

9. Pepinsky RB, Zeng C, Wen D, et al: Identification of a palmitic acid-modified form of human Sonic hedgehog. J Biol Chem 273: 14037-14045, 1998.

10. Porter JA, Young KE and Beachy PA: Cholesterol modification of hedgehog signaling proteins in animal development. Science 274: 255-259, 1996.

11. Kinzler KW, Bigner SH, Bigner DD, et al: Identification of an amplified, highly expressed gene in a human glioma. Science 236: 70-73, 1987.

12. Corbit KC, Aanstad P, Singla V, Norman AR, Stainier DY and Reiter JF: Vertebrate Smoothened functions at the primary cilium. Nature 437: 1018-1021, 2005.

13. Huangfu D and Anderson KV: Cilia and Hedgehog responsiveness in the mouse. Proc Natl Acad Sci USA 102: 11325-11330, 2005.

14. Ruiz i Altaba A, Mas C and Stecca B: The Gli code: an information nexus regulating cell fate, stemness and cancer. Trends Cell Biol 17: 438-447, 2007.

15. Kenney AM, Cole MD and Rowitch DH: Nmyc upregulation by sonic hedgehog signaling promotes proliferation in developing cerebellar granule neuron precursors. Development 130: 15-28, 2003.

16. Kasper M, Schnidar H, Neill GW, et al: Selective modulation of Hedgehog/GLI target gene expression by epidermal growth factor signaling in human keratinocytes. Mol Cell Biol 26: 6283-6298, 2006.

17. Yoon JW, Kita Y, Frank DJ, et al: Gene expression profiling leads to identification of GLI1-binding elements in target genes and a role for multiple downstream pathways in GLI1-induced cell transformation. J Biol Chem 277: 5548-5555, 2002.

18. Teh MT, Blaydon D, Chaplin T, et al: Genomewide single nucleotide polymorphism microarray mapping in basal cell carcinomas unveils uniparental disomy as a key somatic event. Cancer Res 65: 8597-8603, 2005

19. Eichberger T, Kaser A, Pixner C, et al: GLI2-specific transcriptional activation of the bone morphogenetic protein/activin antagonist follistatin in human epidermal cells. J Biol Chem 283: 12426-12437, 2008.

20. Kump E, Ji J, Wernli M, Häusermann P and Erb P: Gli2 upregulates cFlip and renders basal cell carcinoma cells resistant to death ligand-mediated apoptosis. Oncogene 27: 3856-3864, 2008.

21. Hallikas O, Palin K, Sinjushina N, et al: Genome-wide prediction of mammalian enhancers based on analysis of transcription-factor binding affinity. Cell 124: 47-59, 2006.

22. Li X, Deng W, Lobo-Ruppert SM and Ruppert JM: Gli1 acts through Snail and E-cadherin to promote nuclear signaling by $\beta$-catenin. Oncogene 26: 4489-4498, 2007.

23. Kasper M, Regl G, Frischauf AM and Aberger F: GLI transcription factors: mediators of oncogenic Hedgehog signalling. Eur J Cancer 42: 437-445, 2006.

24. Fernandez-Zapico ME: Primers on molecular pathways GLI: more than just Hedgehog? Pancreatology 8: 227-229, 2008.

25. Stecca B, Mas C, Clement V, et al: Melanomas require HEDGEHOG-GLI signaling regulated by interactions between GLI1 and the RAS-MEK/AKT pathways. Proc Natl Acad Sci USA 104: 5895-5900, 2007.

26. Kudchadkar R, Lewis $K$ and Gonzalez R: Advances in the treatment of Basal cell carcinoma: Hedgehog inhibitors. Semin Oncol 39: 139-144, 2012.

27. Wang X, Venugopal C, Manoranjan B, et al: Sonic hedgehog regulates Bmil in human medulloblastoma brain tumor-initiating cells. Oncogene 31: 187-199, 2012.

28. Singh S, Wang Z, Liang Fei D, et al: Hedgehog-producing cancer cells respond to and require autocrine Hedgehog activity. Cancer Res 71: 4454-4463, 2011. 
29. Saqui-Salces $M$ and Merchant JL: Hedgehog signaling and gastrointestinal cancer. Biochim Biophys Acta 1803: 786-795, 2010.

30. Chung MK, Kim HJ, Lee YS, et al: Hedgehog signaling regulates proliferation of prostate cancer cells via stathmin1. Clin Exp Med 10: 51-57, 2010.

31. Tian H, Callahan CA, DuPree KJ, et al: Hedgehog signaling is restricted to the stromal compartment during pancreatic carcinogenesis. Proc Natl Acad Sci USA 106: 4254-4259, 2009.

32. Dierks C, Grbic J, Zirlik K, et al: Essential role of stromally induced hedgehog signaling in B-cell malignancies. Nat Med 13: 944-951, 2007.

33. Becher OJ, Hambardzumyan D, Fomchenko EI, et al: Gli activity correlates with tumor grade in platelet-derived growth factorinduced gliomas. Cancer Res 68: 2241-2249, 2008.

34. Romer JT, Kimura H, Magdaleno S, et al: Suppression of the Shh pathway using a small molecule inhibitor eliminates medulloblastoma in $\mathrm{Ptcl}^{+/-}$p53-/- mice. Cancer Cell 6: 229-240, 2004.

35. Gailani MR, Ståhle-Bäckdahl M, Leffell DJ, et al: The role of the human homologue of Drosophila patched in sporadic basal cell carcinomas. Nat Genet 14: 78-81, 1996

36. Watkins DN, Berman DM, Burkholder SG, Wang B, Beachy PA and Baylin SB: Hedgehog signalling within airway epithelial progenitors and in small-cell lung cancer. Nature 422: 313-317, 2003.

37. Lauth M, Bergström A, Shimokawa T, et al: DYRK1B-dependent autocrine-to-paracrine shift of Hedgehog signaling by mutant RAS. Nat Struct Mol Biol 17: 718-725, 2010.

38. Katano M: Hedgehog signaling pathway as a therapeutic target in breast cancer. Cancer Lett 227: 99-104, 2005.

39. Hatsell S and Frost AR: Hedgehog signaling in mammary gland development and breast cancer. J Mammary Gland Biol Neoplasia 12: 163-173, 2007.

40. Kasper M, Jaks V, Fiaschi M and Toftgård R: Hedgehog signalling in breast cancer. Carcinogenesis 30: 903-911, 2009.

41. Wang LH, Choi YL, Hua XY, et al: Increased expression of sonic hedgehog and altered methylation of its promoter region in gastric cancer and its related lesions. Mod Pathol 19: 675-683, 2006.

42. Varnat F, Duquet A, Malerba M, et al: Human colon cancer epithelial cells harbour active HEDGEHOG-GLI signalling that is essential for tumour growth, recurrence, metastasis and stem cell survival and expansion. EMBO Mol Med 1: 338-351, 2009.

43. Mazumdar T, DeVecchio J, Agyeman A, Shi T and Houghton JA The GLI genes as the molecular switch in disrupting Hedgehog signaling in colon cancer. Oncotarget 2: 638-645, 2011.

44. Wang WS, Chen PM and Su Y: Colorectal carcinoma: from tumorigenesis to treatment. Cell Mol Life Sci 63: 663-671, 2006

45. Theunissen JW and de Sauvage FJ: Paracrine Hedgehog signaling in cancer. Cancer Res 69: 6007-6010, 2009.

46. Lu JT, Zhao WD, He W and Wei W: Hedgehog signaling pathway mediates invasion and metastasis of hepatocellular carcinoma via ERK pathway. Acta Pharmacol Sin 33: 691-700, 2012.

47. Wang Y, Han C, Lu L, Magliato S and Wu T: Hedgehog signaling pathway regulates autophagy in human hepatocellular carcinoma cells. Hepatology: Mar 16, 2013 (Epub ahead of print).

48. Sicklick JK, Li YX, Jayaraman A, et al: Dysregulation of the Hedgehog pathway in human hepatocarcinogenesis. Carcinogenesis 27: 748-757, 2006.

49. Tada M, Kanai F, Tanaka Y, et al: Down-regulation of hedgehoginteracting protein through genetic and epigenetic alterations in human hepatocellular carcinoma. Clin Cancer Res 14: 3768-3776, 2008

50. Chan IS, Guy CD, Chen Y, et al: Paracrine Hedgehog signaling drives metabolic changes in hepatocellular carcinoma. Cancer Res 72: 6344-6350, 2012.
51. Arzumanyan A, Sambandam V, Clayton MM, et al: Hedgehog signaling blockade delays hepatocarcinogenesis induced by hepatitis B virus X protein. Cancer Res 72: 5912-5920, 2012.

52. Pereira Tde A, Witek RP, Syn WK, et al: Viral factors induce Hedgehog pathway activation in humans with viral hepatitis, cirrhosis, and hepatocellular carcinoma. Lab Invest 90 $1690-1703,2010$

53. Philips GM, Chan IS, Swiderska M, et al: Hedgehog signaling antagonist promotes regression of both liver fibrosis and hepatocellular carcinoma in a murine model of primary liver cancer. PLoS One 6: e23943, 2011.

54. Chen X, Lingala S, Khoobyari S, Nolta J, Zern MA and Wu J: Epithelial mesenchymal transition and hedgehog signaling activation are associated with chemoresistance and invasion of hepatoma subpopulations. J Hepatol 55: 838-845, 2011.

55. Cooper MK, Porter JA, Young KE and Beachy PA: Teratogenmediated inhibition of target tissue response to Shh signaling. Science 280: 1603-1607, 1998.

56. Rubin LL and de Sauvage FJ: Targeting the Hedgehog pathway in cancer. Nat Rev Drug Discov 5: 1026-1033, 2006.

57. Robarge KD, Brunton SA, Castanedo GM, et al: GDC-0449-a potent inhibitor of the hedgehog pathway. Bioorg Med Chem Lett 19: 5576-5581, 2009.

58. Yauch RL, Gould SE, Scales SJ, et al: A paracrine requirement for hedgehog signalling in cancer. Nature 455: 406-410, 2008

59. Tian F, Mysliwietz J, Ellwart J, Gamarra F, Huber RM and Bergner A: Effects of the Hedgehog pathway inhibitor GDC-0449 on lung cancer cell lines are mediated by side populations. Clin Exp Med 12: 25-30, 2012.

60. Ailles L and Siu LL: Targeting the Hedgehog pathway in cancer: can the spines be smoothened? Clin Cancer Res 17: 2071-2073, 2011.

61. Singh BN, Fu J, Srivastava RK and Shankar S: Hedgehog signaling antagonist GDC-0449 (Vismodegib) inhibits pancreatic cancer stem cell characteristics: molecular mechanisms. PLoS One 6: e27306, 2011.

62. Von Hoff DD, LoRusso PM, Rudin CM, et al: Inhibition of the hedgehog pathway in advanced basal-cell carcinoma. N Engl J Med 361: 1164-1172, 2009.

63. De Smaele E, Ferretti E and Gulino A: Vismodegib, a smallmolecule inhibitor of the hedgehog pathway for the treatment of advanced cancers. Curr Opin Investig Drugs 11: 707-718, 2010.

64. Rudin CM: Vismodegib. Clin Cancer Res 18: 3218-3222, 2012.

65. Axelson M, Liu K, Jiang X, et al: U.S. Food and Drug Administration approval: vismodegib for recurrent, locally advanced, or metastatic basal cell carcinoma. Clin Cancer Res 19: 2289-2293, 2013.

66. Skvara H, Kalthoff F, Meingassner JG, et al: Topical treatment of Basal cell carcinomas in nevoid Basal cell carcinoma syndrome with a smoothened inhibitor. J Invest Dermatol 131: 1735-1744, 2011.

67. Li Y, Maitah MY, Ahmad A, Kong D, Bao B and Sarkar FH: Targeting the Hedgehog signaling pathway for cancer therapy. Expert Opin Ther Targets 16: 49-66, 2012.

68. Lauth M, Bergström A, Shimokawa T and Toftgård R: Inhibition of GLI-mediated transcription and tumor cell growth by smallmolecule antagonists. Proc Natl Acad Sci USA 104: 8455-8460, 2007.

69. Fu J, Rodova M, Roy SK, et al: GANT-61 inhibits pancreatic cancer stem cell growth in vitro and in NOD/SCID/IL2R gamma null mice xenograft. Cancer Lett 330: 22-32, 2013. 